\title{
The influence and interaction of tobacco-related information exposure on youth smoking behavior and smoking susceptibility:
}

\section{sex and school type difference}

Author:

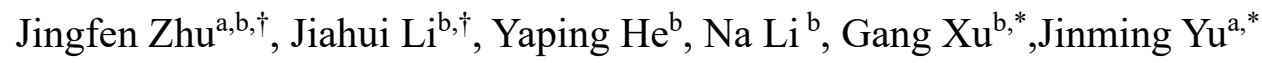

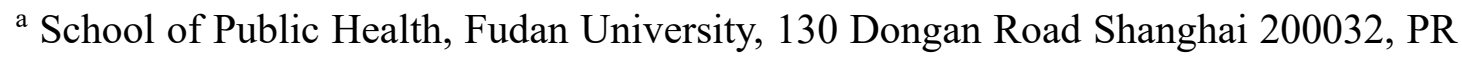
China

${ }^{\mathrm{b}}$ School of Public Health, Shanghai Jiao Tong University, 227 South Chongqing Road, Shanghai 200025, PR China

$\uparrow$ These authors contributed equally to this work.

* Corresponding author :

G Xu, E-mail address: xugang567@sina.com

J Yu, E-mail address: jmy@fudan.edu.cn.

\begin{abstract}
This study investigated the influence and interaction of tobacco promotional and control information with adolescents' current smoking and smoking susceptibility. 12,278 students were recruited from junior, senior and vocational high schools located in Shanghai, China. The exposure to tobacco promotional and control message of participants over the past 30 days were examined, as well as current smoking and never smokers' initiation susceptibility. Complex sample analysis was applied. Descriptive and multivariate logistic regressions were conducted. 89.3\% and $91.5 \%$ of adolescents investigated were exposed to tobacco promotional and control message respectively, which separately increased and decreased the risk of current smoking and never-smokers' smoking susceptibility, especially among males and junior high school students. The risk changed in consistency with the exposure level $\left(P_{\text {trend }}<0.001\right)$. Tobacco control message seemed to mitigate the influence of tobacco promotions in the risk of both current smoking ( $\mathrm{OR}=0.64,95 \% \mathrm{CI}$ : $0.41-0.99)$ and smoking susceptibility ( $\mathrm{OR}=0.65,95 \% \mathrm{CI}$ : 0.46-0.93). Tobacco-related message exposure was highly prevalent and associated with youth smoking risk and smoking susceptibility. It is important to enhance the comprehensiveness and enforcement of
\end{abstract}


promotion bans. Given the improvement of tobacco control message on smoking risk brought by tobacco promotions, the publicity and dissemination of tobacco control information need to be consistently strengthened.

Key words: adolescent; current smoking; smoking behavior; tobacco message exposure

\section{Introduction}

Tobacco use is one of the major preventable causes of death in the world. It is predicted that tobacco use will lead to more than 8 million deaths every year by 2030[1]. As the largest tobacco consuming country, China is also faced with a serious smoking problem [2]. It is of concern that adolescence is the vulnerable period of tobacco addiction and the age of smoking initiation has been decreasing in recent years [2].Scientific evidences showed that youth smoking could cause serious damage to their respiratory and cardiovascular systems, and even accelerate the occurrence of chronic diseases in adulthood[3].

Previous studies have investigated contributors to adolescent smoking. Apart from personal factors (such as sex, age, stress and pocket money), family environment (such as parental smoking) and school environment (peer smoking) [4], social environment is also an important factor which affects youth smoking behaviors. Studies have confirmed [5] that environmental tobacco smoke exposure was a significant predictor of adolescents' smoking habits. Besides, media exposure is also a non-negligible factor, whether it is tobacco promotion or control advertising, considering that adolescents are in a rapidly developing media age now.

The 2012 US Surgeon General's Report determined a causal association between tobacco promotional advertising and adolescents' smoking initiation and progression [1]. The marketing strategies of tobacco companies include indirect methods, such as sponsorship in sports events and concerts, as well as direct methods, like billboards and commercials [6]. It was noted that adolescents were commonly exposed to 
tobacco promotional advertising according to previous studies. For example, In Northern Africa [7], there were $90 \%$ of adolescents investigated reported seeing smoking on the screen, $40 \%$ and $50 \%$ reported seeing a smoking advertisement at live event or in a magazine respectively. In addition to traditional forms of tobacco advertising, there has been an increase in advertising exposure on social media sites [8]. Researches have revealed that young people [9]were regularly exposed to Internet-based tobacco advertising through texts, images, and videos [8]. These advertising and promotions not only influence adolescents' perceived norms, perceived smoking prevalence, and perceptions about smoking benefits and damage[6], but also indirectly influence adolescents' smoking risk by affecting the important others' smoking habits such as parents and peers[7]. The positive association between tobacco advertising and youth smoking behavior has been well established in previous studies [8, 10].

In order to combat the harmful effects of tobacco promotions on adolescents, there were various anti-tobacco advertisements emerging. The themes of most advertisements emphasize adverse health effects, resisting social pressures and influences, or the profitability means of tobacco industries [11]. A number of studies $[12,13]$ examining the impact of anti-tobacco media reported the reduction or prevention of tobacco use among youth. Nevertheless, the interaction between proand anti-tobacco message exposure remained uncertain. Straub and colleagues (2003)found that anti-tobacco advertisement had some preventive effects but could not completely offset the harmful impact of pro-tobacco advertisements[11]; however, there was also study [14]indicating that tobacco control advertising may counteract part of the effectiveness of cigarette advertising in promoting adolescent smoking behavior.

Since adolescents are most likely exposed to the effects of both pro- and antitobacco advertising simultaneously [15], studying their interaction is obviously very important. To date, there are few such researches conducted in China. To our knowledge, our study was the first to examine the effects of exposure to both pro- and anti-smoking media on smoking susceptibility and current smoking beside their 
independent impact. In addition, our study innovatively classified exposure levels into low, medium and high, and compared the differences between sexes and school types. Although China has put efforts on banning tobacco advertising and promoting tobacco control messages, the importance and effectiveness of tobacco control advertising has rarely been proven. Our study explored whether tobacco control advertising counteracts influence of receptivity to cigarette advertising. Findings of our study may inform policy and resource decisions and contribute to more effective tobacco control programs.

\section{Methods}

\subsection{Procedure}

Written informed consent was obtained from all recruited students, their guardians, and school organizers provided before enrollment. The informed consent covered objectives, procedures, potential risks and benefits of the study. The study was approved by the Ethics Committee of the School of Public Health, Shanghai Jiao Tong University. This cross-sectional study was conducted in September 2017 through multistage and stratified cluster random sampling. Participants were adolescents attending junior, senior and vocational high schools in Shanghai. All districts in Shanghai were stratified into urban and suburb areas; four districts were randomly selected, with Huangpu and Putuo as urban areas, Minhang and Jiading as suburb areas. All schools in these four districts were further stratified into junior high schools, senior high schools, and vocational high schools. From these, a total of 33 schools were randomly selected, with 1 vocational school chosen randomly from each district. Participation in this study was completely voluntary. Of the 12,422 adolescents who participated, 12,278(98.8\%) completed the investigation and were included in the analysis.

\subsection{Measures}

The questionnaire used in this study was revised on the basis of Global Youth Tobacco Survey (GYTS) and was proved of sufficient validity and reliability [7]. 


\subsubsection{Dependent variables}

Participants were asked by the question 'have you ever tried cigarette smoking (even one or two puffs)?' and 'on how many days have you smoked in the past 30 days?' Those who reported smoking more than one day in the past 30 days were classified as current smokers and those who reported lifetime smoking but haven't smoked in the past 30 days were classified as ever smokers. All others were classified as never smokers.[16]

Never-smokers were asked two validated questions to determine their susceptibility to smoking in the future [1]:'Do you think you will smoke a cigarette sometime in the next 12 months?' and 'If your best friend offered you a cigarette, would you try it?' (Response options: 1=definitely no, 2=probably no, 3=probably yes, $4=$ definitely yes). Participants were classified as susceptible if they didn't answer 'definitely no' to both questions, and the rest were classified as non-susceptible.

\subsubsection{Independent variables}

Exposure to anti-tobacco message was measured by the following questions: Have you noticed any information describing the dangers of smoking in the following channels and places respectively within the past 30 days:(a)traditional media (such as newspapers/magazines, television, films, broadcasting, notice boards),(b)internet media (such as cell phone, computers/WeChat, digital magazines),(c)mobile digital billboards on buses or subways, (d)billboards in living quarters(such as supermarket or shop store)?, (e)sports events, exhibitions, concerts, community assembly, or community activities?

Exposure to pro-tobacco message was also measured by the above questions, except that the exposed message was the brand or tobacco advertisement, and one more question: 'When you watch TV, video or movies, have you seen actors smoking?' Answer options included a) 'I have no contact with such situations'b) 'I have always seen it (more than 4 times) 'c) 'I have sometimes seen it (1-3 times)' d) 'I have never seen it'. Those who answered 'no contact' or 'never seen'scored 0 , those who 
answered 'sometimes seen'scored 1, and those who answered 'always seen'scored 2. We calculated the total scores and classified students as 'no exposure' if the total score was 0 and others were classified as 'exposure'. Considering two types of exposure, adolescents were categorized to: (a) only anti-tobacco message exposure, (b) only pro-tobacco message exposure, (c) both or (d) neither. In order to investigate the effects of different exposure levels, we trisected the exposure level. Students were classified as 'high level', 'medium level' and 'low level' if the total score ranged between 8-10, 5-7, 0-4 for anti-tobacco message exposure and 5-12, 2-4, 0-1 for pro-tobacco message exposure respectively.

\subsubsection{Covariates}

Participants reported their sex, age, grade level, school type (junior, senior, or vocational high schools), residence (local or non-local), boarding situation (boarder or extern), GPA (top $25 \%$, average, bottom $25 \%$ or unknown), monthly pocket money ( $<200 \mathrm{RMB}, 200-600 \mathrm{RMB}$ or $\geq 600 \mathrm{RMB}$ ), and school district(urban area or suburb area).

Students were asked by two separate questions: 'Does your father smoke?' and 'Does your mother smoke? '[17]Parents' smoking was classified into three categories: 'none or don't know', 'one parent smokes' and 'both parents smoke'. The item 'Do you have friends who smoke?' assessed friends' smoking and classified it into three categories as: 'none', 'some of them' and 'majority or all of them'.

Depressive state was measured by Patient Health Questionnaire 2-item Chinese Version (PHQ-2-C), which assessed changes in interest and mood over the past two weeks. Previous study[18] proved that PHQ-2 score $\geq 3$ had a sensitivity of $74 \%$ and specificity of $75 \%$ for detecting adolescent meeting DSM-IV criteria for major depression on the DISC-IV, and a sensitivity of $96 \%$ and a specificity of $82 \%$ for detecting adolescent meeting criteria for probable major depression on the PHQ-9. The research among Chinese adolescents[19] also indicated a good sensitivity (88\%-94\%) and specificity (73\% 76\%) of PHQ-2. The PHQ-2 is comprised of two items: 'Having little interest or pleasure in doing things' and 'feeling down, depressed, or hopeless'. Scores for each item range from 0 'not at all' to 3 'nearly every day' 
with a total score ranging from 0 to 6 . Participants with score $\geq 3$ were considered as depressed [18].

\subsection{Data Analysis}

To take the complex survey sample design into account, we used Statistical Package for the Social Sciences (SPSS, IBM) version 22.0 for all statistical analyses. Sample characteristics and smoking related characteristics were summarized using weighted percentages and confidence intervals. Percentage and chi-square test were used to compare tobacco message exposure between different sexes and different types of school. Adjusted odds ratios (AORs) with 95\% confidence intervals (CIs) of the associations between tobacco message exposure level and current smoking as well as smoking susceptibility were estimated by multivariate logistic regression models after calibrating covariates at baseline and stratifying by sex and school type. Multivariate logistic regression was used because it accounts for several confounding variables simultaneously. A p value $<0.05$ was considered statistically significant.

\section{Results}

\subsection{Descriptive statistics}

The overall sample was composed of 12,278 students attending junior high school (61.99\%), senior high school (23.67\%), or vocational high school (14.34\%) in Shanghai. As shown in Table1, approximately half (51.60\%) of the participants were male. The majorities of students were local residents $(72.21 \%)$ and externs(86.45\%). More than half of students had monthly pocket money of less than 200 RMB (61.23\%), and an average GPA or below (59.73\%). In addition, over half of them had at least one parent as a smoker (63.44\%). Less than $20 \%$ of them had friends as smokers (17.21\%). More than $10 \%$ of students were classified as depressed (12.45\%). As for smoking status, $92.12 \%$ of students were never smokers, $5.36 \%$ were ever smokers and $2.52 \%$ were current smokers. Nearly $8 \%$ of students showed susceptibility to smoke in the future $(7.67 \%)$. 
Table1 Characteristics distribution and tobacco use among adolescents in Shanghai, China

\begin{tabular}{|c|c|c|c|c|c|}
\hline & & \multicolumn{3}{|c|}{ Weighted } & \multirow{2}{*}{$\begin{array}{l}\text { Unweighted } \\
\mathrm{N}(\%)\end{array}$} \\
\hline & & $\%$ & $95 \% \mathrm{CI}$ & Number & \\
\hline \multicolumn{2}{|l|}{ Age $($ mean, $95 \% \mathrm{CI})$} & 14.28 & $14.23-14.31$ & 670050 & $14.62(14.60-14.97)$ \\
\hline \multirow[t]{3}{*}{ Type of school } & Junior high school & 61.99 & $61.13-62.85$ & 415377 & $6462(52.63)$ \\
\hline & Senior high school & 23.67 & $22.88-24.48$ & 158593 & $2475(20.16)$ \\
\hline & Vocational high school & 14.34 & $13.86-14.83$ & 96080 & $3341(27.21)$ \\
\hline \multirow[t]{2}{*}{ District } & Urban & 33.36 & $33.13-33.60$ & 223534 & $4042(32.92)$ \\
\hline & Suburbs & 66.64 & $66.40-66.87$ & 446516 & $8236(67.08)$ \\
\hline \multirow[t]{2}{*}{ Sex } & Male & 51.60 & $50.69-52.52$ & 345778 & $6419(52.28)$ \\
\hline & Female & 48.40 & $47.48-49.31$ & 324272 & $5859(47.72)$ \\
\hline \multirow[t]{2}{*}{ Residence } & Local & 72.21 & $71.39-73.02$ & 483864 & $8755(71.31)$ \\
\hline & Non-local & 27.79 & $26.98-28.61$ & 186186 & $3523(28.69)$ \\
\hline \multirow[t]{2}{*}{ Boarding in school } & Yes & 13.55 & $13.00-14.12$ & 90791 & $2302(18.75)$ \\
\hline & No & 86.45 & $85.88-87.00$ & 579260 & $9976(81.25)$ \\
\hline \multirow[t]{3}{*}{ Monthly allowance } & $<200 \mathrm{RMB}$ & 61.23 & $60.35-62.11$ & 173882 & $7011(57.10)$ \\
\hline & 200-600RMB & 25.95 & $25.17-26.75$ & 85865 & $3401(27.70)$ \\
\hline & $\geq 600 \mathrm{RMB}$ & 12.81 & $12.24-13.41$ & 670050 & $1866(15.20)$ \\
\hline \multirow[t]{4}{*}{ GPA } & Top 25\% & 32.96 & $32.11-33.83$ & 220860 & $3924(31.96)$ \\
\hline & Average & 46.28 & $45.38-47.20$ & 310129 & $5712(46.52)$ \\
\hline & Bottom 25\% & 13.45 & $12.84-14.09$ & 90136 & $1645(13.40)$ \\
\hline & Not sure & 7.30 & $6.85-7.78$ & 48925 & $997(8.12)$ \\
\hline \multirow[t]{3}{*}{ Parents' smoking } & None & 36.56 & $35.68-37.44$ & 244962 & $4377(35.65)$ \\
\hline & Some & 59.25 & $58.35-60.14$ & 396985 & 7364(59.98) \\
\hline & Most or all & 4.19 & $3.85-4.57$ & 28103 & $537(4.37)$ \\
\hline \multirow[t]{3}{*}{ Friends' smoking } & None & 82.79 & $82.13-83.43$ & 554740 & $9693(78.95)$ \\
\hline & Some & 15.16 & $14.55-15.79$ & 101550 & $2274(18.52)$ \\
\hline & Most or all & 2.05 & $1.83-2.31$ & 13760 & $311(2.53)$ \\
\hline \multirow[t]{2}{*}{ Depression } & No & 87.55 & $86.94-88.13$ & 586624 & $10688(87.05)$ \\
\hline & Yes & 12.45 & $11.87-13.06$ & 83426 & $1590(12.95)$ \\
\hline \multirow[t]{3}{*}{ Smoking status } & Never & 92.12 & $91.64-92.57$ & 617244 & 11091(90.33) \\
\hline & Ever & 5.36 & $4.98-5.76$ & 35893 & $780(6.35)$ \\
\hline & Current & 2.52 & $2.28-2.80$ & 16913 & $407(3.31)$ \\
\hline \multirow[t]{2}{*}{$\begin{array}{l}\text { Smoking } \\
\text { susceptibility }\end{array}$} & No & 92.33 & $91.86-92.78$ & 618651 & $11140(90.73)$ \\
\hline & Yes & 7.67 & $7.22-8.14$ & 51399 & $1138(9.27)$ \\
\hline
\end{tabular}

\subsection{Exposure to anti-tobacco and pro-tobacco messages}

As shown in Table 2, the overall exposure rates of pro-tobacco and anti-tobacco messages were $89.3 \%$ and $91.5 \%$ respectively. After classifying the exposure levels, urban school students were more likely to be exposed to high-level anti-tobacco 
messages than suburb school students $(42.3 \%$ versus $36.7 \%, \mathrm{P}<0.001)$, while female more likely than male students $(39.9 \%$ versus $37.2 \%, \mathrm{P}<0.001)$, and junior high school more likely than senior high school students $(42.3 \%$ versus $33.3 \% \& 31.0 \%$, $\mathrm{P}<0.001)$. With regard to high-level pro-tobacco message, the results showed the opposite: suburb school students were more likely to be exposed than urban school students $(37.5 \%$ versus $33.3 \%, \mathrm{P}<0.001)$, male more than female students $(36.5 \%$ versus $35.7 \%, \mathrm{P}=0.007$ ), and vocational school students were the most likely to be exposed, followed by senior and then junior high school students $(46.1 \%$ versus $37.4 \% \& 33.3 \%, \mathrm{P}<0.001)$.

\subsection{The impact of anti- and pro-tobacco message exposure}

Findings from multiple logistic regression revealed that compared to low anti-tobacco message exposure, those who reported moderate exposure $(\mathrm{OR}=0.60,95 \%$ CI: 0.44 to 0.82$)$ or high exposure (OR=0.54, $95 \%$ CI: 0.39 to 0.76$)$ were less likely to become current smokers with statistical significance. Concerning pro-tobacco messages, those who reported moderate exposure $(\mathrm{OR}=1.58,95 \% \mathrm{CI}: 1.10$ to 2.26$)$ or high exposure (OR=1.98, 95\% CI: 1.39 to 2.81$)$ were more likely to become current smokers, compared to low pro-tobacco message exposure. The risk changes in consistent with the exposure level, regardless of the exposure type $\left(\mathrm{P}_{\text {trend }}<0.001\right)$. After stratifying by sex and school type, we found no statistical significance in female students and senior high school students, but the trend remained among male students and junior high school students by level of tobacco message exposure regardless any type, as shown in Table 3.

The same situation held true in smoking susceptibility: among never smokers, those who reported moderate exposure $(\mathrm{OR}=0.50,95 \% \mathrm{CI}: 0.39$ to 0.64$)$ or high exposure $(\mathrm{OR}=0.37,95 \% \mathrm{CI}: 0.28$ to 0.48$)$ to anti-tobacco message were less likely to start smoking, and those who reported moderate exposure $(\mathrm{OR}=1.74,95 \% \mathrm{CI}: 1.31$ to 2.31 ) or high exposure ( $\mathrm{OR}=2.63,95 \% \mathrm{CI}$ : 2.00 to 3.47$)$ to pro-tobacco message had a higher odds of smoking susceptibility, compared to those who were low exposed, as shown in Table 4. This association was observed in both sexes and all school types. 
The odds increased as exposure levels increased $\left(\mathrm{P}_{\text {trend }}<0.001\right)$. After stratifying by sex, we found that this association was stronger in male students than female students when exposed to moderate (OR: 1.69 vs. 1.53) and high (OR: 2.45 vs. 2.30) level of pro-tobacco message. Nevertheless, when exposed to anti-tobacco message, female students (OR: moderate: 0.48; high: 0.34) were more affected than male students (OR: moderate: 0.54 ; high: 0.32). After stratifying by school types, we found that junior high school students were most vulnerable to pro-tobacco message exposure while senior high school students were most susceptible to anti-tobacco message exposure.

\subsection{The interaction between anti-tobacco messages and pro-tobacco messages}

We reported the relationship between pro- and anti-tobacco messages as a combined variable for current smoking as well as smoking susceptibility.

Among current smokers, adolescents exposed to only pro-tobacco messages represented the highest smoking rate $(7.3 \%)$ and we used these as the reference group (Table 3). Teens exposed to both pro- and anti-tobacco messages appeared to be $36 \%$ less likely to become current smokers compared with this reference group statistically significant ( $\mathrm{OR}=0.64,95 \% \mathrm{CI}: 0.41$ to 0.99$)$. Teens exposed to anti-tobacco messages alone were more than half less likely to become current smokers $(\mathrm{OR}=0.48,95 \% \mathrm{CI}$ : 0.23 to 0.98 ). After stratifying by sex and school type, the risks among those who exposed to anti-tobacco messages decreased most significantly among female students (OR=0.40, 95\% CI: 0.20 to 0.79$)$ and junior high school students $(\mathrm{OR}=0.44$, 95\% CI: 0.19 to 1.01 ) when exposed to pro-tobacco messages at the same time.

Among never smokers, adolescents exposed to only pro-tobacco message also represented the highest rate of smoking susceptibility $(7.8 \%)$ and became the reference group (Table 4). Compared to the reference group, teens exposed to both pro- and anti-tobacco message appeared to be $35 \%$ less likely to start smoking (OR=0.65, 95\% CI: 0.46 to 0.93 ), which was most significantly observed in senior high school $(\mathrm{OR}=0.46,95 \% \mathrm{CI}: 0.24$ to 0.86$)$. Teens exposed to anti-tobacco messages only were the least likely to start smoking ( $\mathrm{OR}=0.21,95 \% \mathrm{CI}$ : 0.09 to 0.45$)$, regardless of sex or school type. 
Table 2 Anti-smoking and pro-smoking information distribution among adolescents in different district, sex and school

\begin{tabular}{|c|c|c|c|c|c|c|c|c|c|c|c|c|c|c|}
\hline & \multicolumn{2}{|c|}{ District } & \multirow[b]{2}{*}{$\chi^{2}$} & \multirow[b]{2}{*}{$P$} & \multicolumn{2}{|c|}{ Sex } & \multicolumn{7}{|c|}{ High school } & \multirow[t]{2}{*}{ Total } \\
\hline & Urban & Suburbs & & & Male & Female & $\chi^{2}$ & $P$ & Junior & Senior & Occupation & $\chi^{2}$ & $P$ & \\
\hline \multicolumn{15}{|c|}{ Anti-exposure } \\
\hline No & $8.6(7.7-9.5)$ & $8.5(7.9-9.1)$ & 0.01 & 0.919 & $10.8(10.0-11.6)$ & $6.1(5.6-6.8)$ & 84.03 & $<0.001$ & $7.3(6.7-7.9)$ & $10.0(8.9-11.2)$ & $11.6(10.5-12.7)$ & 24.64 & $<0.001$ & $8.5(8.0-9.0)$ \\
\hline Yes & $91.4(90.5-92.3)$ & $91.5(90.9-92.1)$ & & & $89.2(88.4-90.0)$ & $93.9(93.2-94.4)$ & & & $92.7(92.1-93.3)$ & $90.0(88.8-91.1)$ & $88.4(87.3-89.5)$ & & & $91.5(91.0-92.0)$ \\
\hline \multicolumn{15}{|c|}{ Pro-exposure } \\
\hline No & $10.2(9.3-11.2)$ & $10.1(9.5-10.8)$ & 0.02 & 0.897 & $11.2(10.4-12.0)$ & $9.0(8.3-9.8)$ & 15.76 & $<0.001$ & $10.4(9.7-11.2)$ & $9.3(8.2-10.4)$ & 10.4(9.4-11.4) & 1.83 & 0.164 & $9.6(10.7-89.9)$ \\
\hline Yes & $89.8(88.8-90.7)$ & $89.9(89.2-90.5)$ & & & $88.8(88-89.6)$ & $91(90.2-91.7)$ & & & $89.6(88.8-90.3)$ & $90.7(89.6-91.8)$ & $89.6(88.6-90.6)$ & & & $89.3(90.4-100.0)$ \\
\hline \multicolumn{15}{|c|}{ Anti-exposure } \\
\hline Low & $27.2(25.8-28.6)$ & $30.7(29.7-31.7)$ & 37.06 & $<0.001$ & $32.4(31.2-33.6)$ & $26.4(25.3-27.6)$ & 53.42 & $<0.0012$ & $27.4(26.3-28.5)$ & $32.0(30.2-33.8)$ & $34.7(33.1-36.3)$ & 35.16 & $<0.001$ & $29.5(28.7-30.4)$ \\
\hline Medium & $30.6(29.1-32.0)$ & $32.6(31.6-33.7)$ & & & $30.4(29.2-31.5)$ & $33.6(32.4-34.9)$ & & & $30.3(29.2-31.4)$ & $34.7(32.9-36.6)$ & $34.3(32.8-35.9)$ & & & $31.9(31.1-32.8)$ \\
\hline High & $42.3(40.7-43.8)$ & $36.7(35.6-37.8)$ & & & $37.2(36.0-38.5)$ & $39.9(38.7-41.2)$ & & & $42.3(41.1-43.5)$ & $33.3(31.5-35.2)$ & $31.0(29.5-32.6)$ & & & $38.5(37.7-39.4)$ \\
\hline \multicolumn{15}{|c|}{ Pro-exposure } \\
\hline Low & $38.2(36.7-39.8)$ & $31.7(30.6-32.7)$ & 52.92 & $<0.001$ & $32.6(31.4-33.8)$ & $35.2(34.0-36.5)$ & 10.68 & 0.0073 & $36.4(35.3-37.6)$ & $31.2(29.4-33.0)$ & $27.1(25.6-28.6)$ & 33.86 & $<0.001$ & $33.9(33.0-34.7)$ \\
\hline Medium & $28.5(27.1-30.0)$ & $30.8(29.8-31.8)$ & & & $30.9(29.8-32.1)$ & $29.1(27.9-30.3)$ & & & $30.2(29.1-31.4)$ & $31.4(29.6-33.3)$ & $26.8(25.4-28.4)$ & & & $30(29.2-30.9)$ \\
\hline High & $33.3(31.8-34.8)$ & $37.5(36.5-38.6)$ & & & $36.5(35.3-37.7)$ & $35.7(34.4-36.9)$ & & & $33.3(32.2-34.5)$ & $37.4(35.5-39.3)$ & $46.1(44.4-47.8)$ & & & $36.1(35.2-37)$ \\
\hline
\end{tabular}


Table 3 Media exposure level and current smoking risk of adolescents stratified by sex and school type (OR, 95\% CI) ${ }^{\text {a }}$

\begin{tabular}{|c|c|c|c|c|c|c|c|}
\hline & \multirow{2}{*}{$\begin{array}{l}\text { Current smoking } \\
\%(95 \% \mathrm{CI})\end{array}$} & \multirow{2}{*}{ Total } & \multicolumn{2}{|c|}{ Sex } & \multicolumn{3}{|c|}{ High school } \\
\hline & & & Male & Female & Junior & Senior & Vocational \\
\hline \multicolumn{8}{|l|}{ Anti-exposure } \\
\hline Low & $4.3(3.7-5)$ & $\mathrm{ref}=1$ & $\mathrm{ref}=1$ & $\mathrm{ref}=1$ & $\mathrm{ref}=1$ & $\mathrm{ref}=1$ & $\mathrm{ref}=1$ \\
\hline Medium & $2.1(1.7-2.5)$ & $0.60(0.44-0.82)$ & $0.62(0.43-0.89)$ & $0.61(0.32-1.13)$ & $0.45(0.21-0.96)$ & $0.65(0.29-1.44)$ & $0.81(0.57-1.17)$ \\
\hline High & $1.5(1.2-1.9)$ & $0.54(0.39-0.76)$ & $0.54(0.37-0.80)$ & $0.54(0.28-1.02)$ & $0.41(0.22-0.77)$ & $1.07(0.52-2.21)$ & $0.47(0.31-0.72)$ \\
\hline$P$ for trend & & $<0.001$ & 0.002 & 0.068 & 0.003 & 0.882 & 0.001 \\
\hline \multicolumn{8}{|l|}{ Pro-exposure } \\
\hline Low & $1.5(1.2-1.9)$ & $\mathrm{ref}=1$ & $\mathrm{ref}=1$ & $\mathrm{ref}=1$ & $\mathrm{ref}=1$ & $\mathrm{ref}=1$ & $\mathrm{ref}=1$ \\
\hline Medium & $2.7(2.3-3.3)$ & $1.58(1.10-2.26)$ & $1.92(1.24-2.97)$ & $0.96(0.49-1.87)$ & $2.71(1.17-6.25)$ & $1.51(0.69-3.29)$ & $1.08(0.70-1.66)$ \\
\hline High & $3.3(2.8-3.8)$ & $1.98(1.39-2.81)$ & $2.48(1.62-3.80)$ & $1.17(0.61-2.25)$ & $2.96(1.34-6.56)$ & $1.96(0.82-4.67)$ & $1.44(0.95-2.18)$ \\
\hline$P$ for trend & & $<0.001$ & $<0.001$ & 0.686 & 0.01 & 0.197 & 0.043 \\
\hline \multicolumn{8}{|l|}{ Anti- by pro- exposure } \\
\hline Pro-exposure only & $7.3(5.4-9.7)$ & $\mathrm{ref}=1$ & $\mathrm{ref}=1$ & $\mathrm{ref}=1$ & $\mathrm{ref}=1$ & $\mathrm{ref}=1$ & $\mathrm{ref}=1$ \\
\hline Anti-exposure only & $0.9(0.5-1.7)$ & $0.48(0.23-0.98)$ & $0.41(0.16-1.02)$ & $0.52(0.16-1.73)$ & $0.17(0.03-1.06)$ & $\mathrm{N} / \mathrm{A}$ & $1.69(0.68-4.18)$ \\
\hline Pro- and anti- exposure & $2.2(1.9-2.5)$ & $0.64(0.41-0.99)$ & $0.78(0.45-1.36)$ & $0.40(0.20-0.79)$ & $0.44(0.19-1.01)$ & $0.69(0.32-1.52)$ & $0.99(0.57-1.71)$ \\
\hline No exposure & $7.0(5.1-9.5)$ & $1.00(0.52-1.92)$ & $0.98(0.43-2.20)$ & $1.06(0.33-3.41)$ & $1.03(0.26-4.05)$ & $0.75(0.22-2.57)$ & $1.48(0.69-3.18)$ \\
\hline
\end{tabular}

${ }^{a}$ Model adjusted for age, sex, school type, district, boarding, local, GPA, pocket money, depression motion, friends' smoking and parents' smoking 
Table 4 Media exposure level and smoking susceptibility risk of adolescents stratified by sex and school type among never smokers (OR, $95 \%$ CI) ${ }^{\text {a }}$

\begin{tabular}{|c|c|c|c|c|c|c|c|}
\hline & \multirow{2}{*}{$\begin{array}{l}\text { Susceptibility } \\
\%(95 \% \mathrm{CI})\end{array}$} & \multirow{2}{*}{ Total } & \multicolumn{2}{|c|}{ Sex } & \multicolumn{3}{|c|}{ High school } \\
\hline & & & Male & Female & Junior & Senior & Vocational \\
\hline \multicolumn{8}{|l|}{ Anti-exposure } \\
\hline Low & $2.3(1.9-2.8)$ & $\mathrm{ref}=1$ & $\mathrm{ref}=1$ & $\mathrm{ref}=1$ & ref $=1$ & ref $=1$ & $\mathrm{ref}=1$ \\
\hline Medium & $4.1(3.5-4.8)$ & $0.50(0.39-0.64)$ & $0.54(0.39-0.74)$ & $0.48(0.32-0.71)$ & $0.53(0.35-0.81)$ & $0.38(0.23-0.60)$ & $0.69(0.48-0.98)$ \\
\hline High & $5.4(4.7-6.1)$ & $0.37(0.28-0.48)$ & $0.42(0.31-0.58)$ & $0.34(0.23-0.52)$ & $0.42(0.29-0.62)$ & $0.27(0.16-0.47)$ & $0.45(0.30-0.67)$ \\
\hline$P$ for trend & & $<0.001$ & $<0.001$ & $<0.001$ & $<0.001$ & $<0.001$ & $<0.001$ \\
\hline \multicolumn{8}{|l|}{ Pro-exposure } \\
\hline Low & $2.3(1.9-2.8)$ & $\mathrm{ref}=1$ & ref $=1$ & $\mathrm{ref}=1$ & $\mathrm{ref}=1$ & $\mathrm{ref}=1$ & $\mathrm{ref}=1$ \\
\hline Medium & $4.1(3.5-4.8)$ & $1.74(1.31-2.31)$ & $1.69(1.18-2.42)$ & $1.53(0.98-2.39)$ & $2.20(1.40-3.47)$ & $1.23(0.74-2.04)$ & $1.78(1.12-2.82)$ \\
\hline High & $5.4(4.7-6.1)$ & $2.63(2.00-3.47)$ & $2.45(1.74-3.46)$ & $2.30(1.51-3.52)$ & $3.04(1.93-4.77)$ & $1.95(1.17-3.22)$ & $2.86(1.88-4.34)$ \\
\hline$P$ for trend & & $<0.001$ & $<0.001$ & $<0.001$ & $<0.001$ & 0.017 & $<0.001$ \\
\hline \multicolumn{8}{|l|}{ Anti- by pro- exposure } \\
\hline Pro-exposure only & $7.8(5.7-10.6)$ & $\mathrm{ref}=1$ & $\mathrm{ref}=1$ & ref $=1$ & $\mathrm{ref}=1$ & $\mathrm{ref}=1$ & $\mathrm{ref}=1$ \\
\hline Anti-exposure only & $1.1(0.5-2.1)$ & $0.21(0.09-0.45)$ & $0.28(0.11-0.70)$ & $0.10(0.03-0.36)$ & $0.24(0.06-0.87)$ & $0.18(0.04-0.79)$ & $0.32(0.10-1.03)$ \\
\hline Pro- and anti- exposure & $3.9(3.6-4.3)$ & $0.65(0.46-0.93)$ & $0.67(0.43-1.04)$ & $0.62(0.33-1.16)$ & $0.88(0.48-1.62)$ & $0.46(0.24-0.86)$ & $0.79(0.44-1.43)$ \\
\hline No exposure & $4.2(2.6-6.5)$ & $0.48(0.26-0.90)$ & $0.47(0.22-1.01)$ & $0.53(0.18-1.60)$ & $0.82(0.30-2.25)$ & $0.24(0.07-0.88)$ & $0.48(0.20-1.20)$ \\
\hline
\end{tabular}

${ }^{a}$ Model adjusted for age, sex, school type, district, boarding, local, GPA, pocket money, depression motion, friends' smoking and parents' smoking 


\section{Discussions}

There are few studies on relationship of smoking and tobacco-related message exposure in terms of both exposure level and its mutual influence. To our knowledge, this was the first time such a study conducted in Chinese adolescents. Our study found that the risk of current smoking and never smokers' smoking susceptibility increased as the exposure level of tobacco promotions increased, while decreased when exposed to more tobacco control messages. We also observed a $35 \%$ decrease in both two risks among students receptive to both anti- and pro-tobacco messages compared to those only exposed to pro-tobacco message. Moreover, we found the effects of tobacco-related message exposure were different among different sexes and school types, particularly on current smoking. Our findings could provide theoretical basis and direction for tobacco control in the future.

The exposure of tobacco promotional advertisements to adolescents remains a serious problem over decades, in spite of the release of WHO Framework Convention on Tobacco Control (FCTC) in 2003 which demonstrated the ban on tobacco advertising, promotion and sponsorship[12]. China had ratified the FCTC in 2005, however, the implementation progressed slowly [20]. In many cases, existing policies or regulations were weak due to limited enforcement [21]. Our research showed that overall exposure among youth to pro-tobacco messages is as high as a proportion of $89.3 \%$, which requires much more attention and reflection. Moreover, our study found the situation more serious among male, suburb school and vocational school students, indicating these groups are living in a relatively poor tobacco control environment. These findings were partly attributed to the smoking norms in China that smoking is overwhelmingly a male phenomenon [20]. As a result, tobacco industries mainly target males, and draw them to social environment where tobacco advertising is more prevalent. Higher exposure in suburb school students may be attributed to the differences in tobacco prevalence and environment. These students were involved more in tobacco consumption [22], and suffered more passive smoking [23] for the weak implementation of smoke-free policies in suburb areas, which indicated the 
importance and urgency of tobacco control publicity in the suburbs. Our finding on more exposure among vocational school students could be explained by their special learning and living environment; they enter the workforce directly after graduation and are more likely to attach social smokers[24]. In this case, it was not surprising that they were exposed to a more active tobacco environment. Despite the high exposure level of tobacco promotions, fortunately, adolescents are exposed to an encouraging tobacco control environment as well. Our study found that more than $90 \%$ of adolescents reported having noticed anti-cigarette information during last month, higher than the proportion in Indonesia (71.69\%) [25] and US (57.9\%) [14]. It is undeniable that China has put much effort on tobacco control in recent years. Collectively, these findings suggested that the control of tobacco advertising and the dissemination of tobacco control information still need to be strengthened, especially among male, suburb school and vocational school students.

It is worldwide recognized that pro-tobacco messages, including tobacco advertising and promotions in movies, magazines and other media [26] has an impact on promoting youth smoking. Tobacco industries take advantage of advertisements to arouse adolescents' interest in tobacco use [27], through shaping their perceptions of smoking norm, and perceived benefits and smoking risks [7]. Previous studies[28]showed that exposure to Point-of-sale (POS)promotion was associated with 1.6 and 1.3 times higher odds of experimental smoking and smoking susceptibility respectively among adolescents [10]. Our study has come to the similar conclusions and found that the risks vary with exposure levels. This trend was also observed in a German study[29] that high exposure to cigarette promotions presented a significant predictor of adolescent smoking initiation compared with low exposure $(\mathrm{ARR}=1.46 ; 95 \% \mathrm{CI}: 1.08-1.97)$. One possible explanation could be that adolescents are more interested in tobacco products when exposed to more promotions, which allow them to try smoking and lead to more susceptible to subsequent smoking behaviors[30]. When it comes to tobacco control messages, to which much attention has been paid these years, there were a few studies which have proved that it could reduce youth smoking initiation. Tobacco control advertisements mainly use graphic 
images or individual stories to demonstrate the serious health effects of smoking. Researches [31] found that these advertisements were well accepted by adolescents and attracted them greatly, and thus reduced their smoking intentions. For example, US [13] and Turkey [12] both found that exposure to anti-tobacco advertisements could strengthen youth nonsmoking intentions and perceptions of smoking harm $(\mathrm{OR}=1.25 ; 95 \% \mathrm{CI}: 1.11-1.42)$, and lower odds of becoming a smoker $(\mathrm{OR}=0.74$; 95\%CI: 0.63-0.88) [13]. This correlation has also been confirmed in our study that exposure to tobacco control messages was a protective factor of both current smoking and smoking susceptibility. Since the risk decreased more when adolescents had more exposure, we recommend greater tobacco control message publicity in all sorts of circumstances for more gains.

Sex and school type were stratified for further analysis. In terms of adolescents' current smoking, we found that the association was more pronounced among male and junior high school students, regardless of type of tobacco message exposure. A possible reason could be that the existing tobacco promotion and control information mainly aimed at males, given the fact that this population has a dominant smoking rate [32]. Therefore, both increasing the anti-tobacco and reducing the pro-tobacco message exposure could have considerable benefits on male students. For junior high school students, their immature physiology and psychology for their young age may result in a more sensitivity to both tobacco promotions and tobacco control information. Among all populations of never smokers, the association between tobacco-related messages and smoking susceptibility was more significant. Since smoking initiation was one step to regular smoking [25], the significance of preventing youth from smoking the first cigarette is particularly important. Considering that both the reduction of tobacco promotional advertising and the increase of tobacco control messages could reduce never smokers' intention to smoke, future tobacco control efforts may focus on the regulations on tobacco-related information.

In addition, we also studied the interaction between tobacco control and promotional information. Previous related studies were few [11, 14, 15], and the 
association was not sure till now. There are two general opinions currently, one is that anti-tobacco message was unable to counteract the effects of tobacco advertising in spite of its independent protective effect[15], another is that exposure to anti-tobacco message simultaneously could reduce the probability of smoking initiation among adolescents already exposed to tobacco advertising[14]. Our findings supported the latter opinion that tobacco control message may counter the effectiveness of receptivity to cigarette advertising, with a more than $30 \%$ reduction on risk of both current smoking and smoking susceptibility, suggesting an important window of opportunity to offset the impact of tobacco promotions and advertising. Themes of tobacco control message commonly include adverse health effects of smoking and second hand smoke, tobacco industry manipulation, and the social unacceptability of smoking[33]. These messages make youth less receptive to cigarette advertising, and therefore reduce their smoking willingness and impulse purchase, which may progress into more intensive and frequent smoking. In addition, our study found that among never smokers, the risk of smoking susceptibility decreased most when exposed to anti-tobacco message only, regardless of sex or school type, for a more than $70 \%$ reduction. In order to effectively prevent non-susceptible adolescents from becoming susceptible and reduce smoking prevalence, it is important to increase the publicity of tobacco control information in the case that promotion information cannot be banned completely.

China has ratified the FCTC and published Advertising Law of the People's Republic of China which have clear restrictions and regulations on tobacco advertising for more than a decade; however, it has not been effectively implemented. It is proved [1] that laws enforcing a ban comprehensively on direct and indirect tobacco advertising are effective in reducing tobacco use. We endorse the need for government to assume responsibility for tobacco control, and to enforce the national smoke-free law more powerfully. Besides traditional media, Internet platforms and social media have become one of the new channels through which tobacco industry promotes its products. The present online advertisements are targeting young people with no experience of smoking [8]. In order to reduce the smoking rate and form the 
non- regularization of smoking among youth, online advertising and promotions need more comprehensive restrictions. Nevertheless, it is encouraging to see an inspiring tobacco control environment that most adolescents have been exposed to tobacco control messages. In this context, exploring effective methods and forms of tobacco control messages targeting adolescents has become more and more important. Future studies should focus on developing specific tobacco control advertisements for adolescents. For example, considering releasing messages through new media such as the Internet to gain more attention from adolescents. In addition to social environment, school is also a great platform for tobacco control message exposure. Schools can increase the exposure among students through course education, video broadcasting, posters and other means, so as to form a positive tobacco control environment and reduce students' smoking risk.

This study also has several limitations. First, our cross-sectional research design precluded causal inference. Longitudinal studies are needed to examine causal relationships between tobacco-related message exposure and smoking behaviors. Second, the respondents in our study were adolescents in urban areas of China, thus our results may not reflect rural areas. The third limitation was the indirect gathering of data on tobacco-related message exposure as well as adolescents' smoking behavior and smoking susceptibility based on their own reports. However, studies[34] have confirmed the validity of adolescents' self-reported data. Despite these limitations, this study has some strength. First, we analyzed the exposure level of tobacco-related message and the interaction between different exposures, adjusting for several influencing factors, such as characteristics, psychological factors and parental smoking, to more objectively reflect the impact. Second, we put Internet source into consideration to fully cover the advertising exposure. Future studies investigating how well tobacco control advertising counteracts the effectiveness of cigarette advertising are needed.

\section{Conclusions}

In conclusion, exposure to tobacco promotional and control messages was 
common among adolescents, which associated with their smoking susceptibility and behaviors. The effect changes in consistent with exposure levels. The comprehensiveness and enforcement of bans on tobacco promotions and advertising need to be implemented. Since tobacco control information can not only reduce the smoking risk independently, but also effectively offset the effects of tobacco advertising, we recommend a wider spread of it. Future studies could explore effective forms and approaches of tobacco control conducted among adolescents.

\section{Author contributions}

$\mathrm{G} \mathrm{Xu}, \mathrm{J}$ Yu were responsible for designing the research, writing the study protocol, and revised this manuscript. $\mathrm{J}$ Zhu and $\mathrm{J}$ Li carried out data analysis, result interpretation, and manuscript preparation. Y He, $\mathrm{Li} \mathrm{Na}$ were responsible for data collection and managing field activities. All authors have read and approved the final manuscript.

\section{Acknowledgments:}

This work was supported by the grants from National Natural Science Foundation of China(71403169) and Shanghai Key Discipline Construction Project in Public Health (15GWZK1002).

\section{Conflicts of interest}

The authors declare no conflict of interest.

\section{References}

1. English, L. M.; Hsia, J.; Malarcher, A., Tobacco advertising, promotion, and sponsorship (TAPS) exposure, anti-TAPS policies, and students' smoking behavior in Botswana and South Africa. Preventive medicine 2016, 91s, S28-S34.

2. Zhang, X.; Li, Y.; Zhang, Q.; Lu, F.; Wang, Y., Smoking and its risk factors in Chinese elementary and middle school students: a nationally representative sample study. Addictive behaviors 2014, 39 (5), 837-841.

3. Wang, Y. N.; Qi, F.; Jia, X. R.; Lin, P.; Liu, H.; Geng, M. Y.; Qu, Y. B.; Li, S. P., Smoking attempt and its influence factors among junior high schoolstudents in Qingdao city. Chin J Public Health 2017, 33 (05), 725-729.

4. Gwon, S. H.; Jeong, S., [Factors Influencing Adolescent Lifetime Smoking and Current Smoking in South Korea: Using data from the 10th (2014) Korea Youth Risk Behavior Web-Based Survey]. Journal of Korean Academy of Nursing 2016, 46 (4), 552-561.

5. Seo, D. C.; Bodde, A. E.; Torabi, M. R., Salient environmental and perceptual correlates of current and established smoking for 2 representative cohorts of Indiana adolescents. The Journal of school health 2009, 79 (3), 98-107. 
6. National Center for Chronic Disease, P.; Health Promotion Office on, S.; Health, Reports of the Surgeon General. In Preventing Tobacco Use Among Youth and Young Adults: A Report of the Surgeon General, Centers for Disease Control and Prevention (US): Atlanta (GA), 2012.

7. Madkour, A. S.; Ledford, E. C.; Andersen, L.; Johnson, C. C., Tobacco advertising/promotions and adolescents' smoking risk in Northern Africa. Tobacco control 2014, 23 (3), 244-252.

8. Dunlop, S.; Freeman, B.; Perez, D., Exposure to Internet-Based Tobacco Advertising and Branding: Results From Population Surveys of Australian Youth 2010-2013. Journal of medical Internet research 2016, 18 (6), e104.

9. Agaku, I. T.; King, B. A.; Dube, S. R., Trends in exposure to pro-tobacco advertisements over the Internet, in newspapers/magazines, and at retail stores among U.S. middle and high school students, 2000-2012. Preventive medicine 2014, 58, 45-52.

10. Shang, C.; Huang, J.; Cheng, K. W.; Li, Q.; Chaloupka, F. J., Global Evidence on the Association between POS Advertising Bans and Youth Smoking Participation. International journal of environmental research and public health 2016, 13 (3).

11. Weiss, J. W.; Cen, S.; Schuster, D. V.; Unger, J. B.; Johnson, C. A.; Mouttapa, M.; Schreiner, W. S.; Cruz, T. B., Longitudinal effects of pro-tobacco and anti-tobacco messages on adolescent smoking susceptibility. Nicotine \& tobacco research : official journal of the Society for Research on Nicotine and Tobacco 2006, 8 (3), 455-465.

12. Erguder, T.; Bilir, N.; Ozcebe, H.; Irmak, H.; Tasti, E.; Ilter, H.; Palipudi, K. M.; Andes, L. J.; Asma, S.; Khoury, R. N.; Talley, B., Exposure to anti- and pro-tobacco advertising, promotions or sponsorships: Turkey, 2008. Global health promotion 2016, 23 (2 Suppl), 58-67.

13. Emery, S.; Wakefield, M. A.; Terry-McElrath, Y.; Saffer, H.; Szczypka, G.; O'Malley, P. M.; Johnston, L. D.; Chaloupka, F. J.; Flay, B., Televised state-sponsored antitobacco advertising and youth smoking beliefs and behavior in the United States, 1999-2000. Archives of pediatrics \& adolescent medicine 2005, 159 (7), 639-645.

14. Emory, K. T.; Messer, K.; Vera, L.; Ojeda, N.; Elder, J. P.; Usita, P.; Pierce, J. P., Receptivity to cigarette and tobacco control messages and adolescent smoking initiation. Tobacco control 2015, 24 (3), 281-284.

15. Straub, D. M.; Hills, N. K.; Thompson, P. J.; Moscicki, A. B., Effects of pro- and anti-tobacco advertising on nonsmoking adolescents' intentions to smoke. The Journal of adolescent health : official publication of the Society for Adolescent Medicine 2003, 32 (1), 36-43.

16. Dunlop, S.; Kite, J.; Grunseit, A. C.; Rissel, C.; Perez, D. A.; Dessaix, A.; Cotter, T.; Bauman, A.; Young, J.; Currow, D., Out of Sight and Out of Mind? Evaluating the Impact of Point-of-Sale Tobacco Display Bans on Smoking-Related Beliefs and Behaviors in a Sample of Australian Adolescents and Young Adults. Nicotine \& tobacco research : official journal of the Society for Research on Nicotine and Tobacco 2015, 17 (7), 761-768.

17. Huang, H. W.; Lu, C. C.; Yang, Y. H.; Huang, C. L., Smoking behaviours of adolescents, influenced by smoking of teachers, family and friends. International nursing review 2014, 61 (2), 220-227.

18. Richardson, L. P.; Rockhill, C.; Russo, J. E.; Grossman, D. C.; Richards, J.; McCarty, C.; McCauley, E.; Katon, W., Evaluation of the PHQ-2 as a brief screen for detecting major depression among adolescents. Pediatrics 2010, 125 (5), e1097-1103.

19. Yang, W. H.; Li, L.; Xiao, L. H.; Peng, F.; Liu, H. H., Screening for Depressive Disorder in Adolescents: Validity ofChinese Version of the Patients Health Questionnaire 2-Item. Chinese 
Journal of Clinical Psychology 2015, 23 (05), 937-943.

20. Yang, T.; Rockett, I. R.; Li, M.; Xu, X.; Gu, Y., Tobacco advertising, environmental smoking bans, and smoking in Chinese urban areas. Drug and alcohol dependence 2012, 124 (1-2), 121-127.

21. Li, Q.; Hyland, A.; O'Connor, R.; Zhao, G.; Du, L.; Li, X.; Fong, G. T., Support for smoke-free policies among smokers and non-smokers in six cities in China: ITC China Survey. Tobacco control 2010, 19 Suppl 2, i40-i46.

22. Sabnis, R.; Sahu, K.; Thakur, D.; Surana, S.; Mazhar, H.; Pandey, S., Urban and rural disparity in tobacco use and knowledge about oral cancer among adolescents: An epidemiological survey on 12 and 15-year school going students. Journal of International Society of Preventive \& Community Dentistry 2016, 6 (Suppl 3), S226-S231.

23. Sun, X. S.; Li, L.; Liu, Y. P., Current situation and influencing factors of passive smoking among primary and middle school students in Xuzhou city. Jiangsu $J$ Prev Med 2018, 29 (06), 729-730.

24. Wu, X., Comparative analysis of levels of awareness about the dangersof tobacco knowledge and smoking status between ordinaryhigh school students and vocational high school students. Chin J of PHM 2017, 33 (02), 247-249.

25. Prabandari, Y. S.; Dewi, A., How do Indonesian youth perceive cigarette advertising? A cross-sectional study among Indonesian high school students. Global health action 2016, 9, 30914.

26. Villanti, A.; Boulay, M.; Juon, H. S., Peer, parent and media influences on adolescent smoking by developmental stage. Addictive behaviors 2011, 36 (1-2), 133-136.

27. Pierce, J. P.; Sargent, J. D.; White, M. M.; Borek, N.; Portnoy, D. B.; Green, V. R.; Kaufman, A. R.; Stanton, C. A.; Bansal-Travers, M.; Strong, D. R.; Pearson, J. L.; Coleman, B. N.; Leas, E.; Noble, M. L.; Trinidad, D. R.; Moran, M. B.; Carusi, C.; Hyland, A.; Messer, K., Receptivity to Tobacco Advertising and Susceptibility to Tobacco Products. Pediatrics 2017, 139 (6).

28. Robertson, L.; Cameron, C.; McGee, R.; Marsh, L.; Hoek, J., Point-of-sale tobacco promotion and youth smoking: a meta-analysis. Tobacco control 2016, 25 (e2), e83-e89.

29. Hanewinkel, R.; Isensee, B.; Sargent, J. D.; Morgenstern, M., Cigarette advertising and teen smoking initiation. Pediatrics 2011, 127 (2), e271-e278.

30. Saito, J.; Yasuoka, J.; Poudel, K. C.; Foung, L.; Vilaysom, S.; Jimba, M., Receptivity to tobacco marketing and susceptibility to smoking among non-smoking male students in an urban setting in Lao PDR. Tobacco control 2013, 22 (6), 389-394.

31. White, V. M.; Durkin, S. J.; Coomber, K.; Wakefield, M. A., What is the role of tobacco control advertising intensity and duration in reducing adolescent smoking prevalence? Findings from 16 years of tobacco control mass media advertising in Australia. Tobacco control 2015, 24 (2), 198-204.

32. Zhi, K.; Huang, J.; Deng, S.; Chen, Y.; Vaughn, M. G.; Qian, Z., Decreased smoking initiation among male youths in China: an urban-rural comparison. International journal of public health 2016, 61 (4), 417-425.

33. Pierce, J. P.; White, V. M.; Emery, S. L., What public health strategies are needed to reduce smoking initiation? Tobacco control 2012, 21 (2), 258-264.

34. Kentala, J.; Utriainen, P.; Pahkala, K.; Mattila, K., Verification of adolescent self-reported smoking. Addictive behaviors 2004, 29 (2), 405-411. 